\title{
Infrared Imaging of Meibomian Glands and Evaluation of the Lipid Layer in Sjögren's Syndrome Patients and Nondry Eye Controls
}

\author{
Kara L. Menzies, ${ }^{1,2}$ Sruthi Srinivasan, ${ }^{1,2}$ C. Lisa Prokopich, ${ }^{2}$ and Lyndon Jones ${ }^{1,2}$ \\ ${ }^{1}$ Centre for Contact Lens Research, University of Waterloo, Waterloo, Ontario, Canada \\ ${ }^{2}$ School of Optometry \& Vision Science, University of Waterloo, Waterloo, Ontario, Canada
}

Correspondence: Sruthi Srinivasan, University of Waterloo, 200 University Avenue West, Waterloo, ON, N2L3G1, Canada;

s2sriniv@uwaterloo.ca.

Submitted: January 1, 2014

Accepted: December 8, 2014

Citation: Menzies KL, Srinivasan S, Prokopich CL, Jones L. Infrared imaging of meibomian glands and evaluation of the lipid layer in Sjögren's syndrome patients and nondry eye controls. Invest Ophthalmol Vis Sci. 2015;56:836-841. DOI:10.1167/ iovs.14-13864
Purpose. The purpose of this study was to evaluate meibomian gland dropout and lipid layer thickness (LLT) in patients with and without Sjögren's syndrome dry eye (SS).

MethoDs. We recruited 11 participants with SS (males/females [M/F], 1:10; mean age = 56.0 \pm 9.1 years) and 10 control subjects without dry eye $(\mathrm{M} / \mathrm{F}, 3: 7$; mean age $=58.5 \pm 4.7$ years) . All participants completed the Ocular Surface Disease Index (OSDI) questionnaire. The LLT was assessed using the Tearscope Plus based on the appearance of the lipid layer. Noninvasive tear break-up time (NITBUT) also was measured. The lower and upper lids were everted, and the meibomian glands were imaged using the infrared camera of the Keratograph 4. A meibomian gland dropout score due to gland loss was obtained. Statistical analysis was conducted using the Mann-Whitney $U$ test and correlations were determined using Spearman rank correlations.

RESULTS. Of the SS participants, 100\% reported ocular and oral dryness symptoms in the AECC questionnaire. The SS group recorded a higher OSDI score (median $=48.00$, interquartile range $[\mathrm{IQR}] 23.0-56.2$ vs. 2.1, IQR 0.0-2.6; $P<0.001$ ), reduced LLT (median $[\mathrm{IQR}]=15.0$ $[15.0-15.0]$ vs. $60.0[45.0-100.0] \mathrm{nm} ; P=0.001$ ), and lower NITBUT (median [IQR] $=3.7$ [2.5-4.2] vs. 9.5 [6.4-17.6] sec; $P<0.001)$ compared to the controls. Digital meibomian gland dropout score (\% dropout) was significantly higher for the SS group (16.0\% [IQR 12.1$40.0 \%$ ] vs. $6.7 \%$ [IQR $1.5-12.7 \%$ ] $P=0.01)$. Subjective meibomian gland dropout score $(0-6$ score) was significantly higher for the SS group (median $[\mathrm{IQR}]=1.5[1.0-4.0]$ vs. $1.0[0.0-$ 1.25]; $P=0.03$ ).

Conclusions. Patients with SS showed higher meibomian gland dropout scores and reduced LLT and NITBUT, which likely contribute to the severe dry eye symptoms reported by SS subjects.

Keywords: Sjögren's syndrome, meibography, tear breakup time, lipid layer, dry eye, MGD

\begin{abstract}
jögren's syndrome (SS) is a disease characterized by $\checkmark$ infiltration of lymphocytes into exocrine glands, including sebaceous, sweat, salivary, and lacrimal glands. ${ }^{1-4}$ Following infiltration, gland atrophy and dysfunction occurs. ${ }^{1,5}$ The disease manifests with moderate-to-severe dry eye and dry mouth, and may occur in combination with other systemic manifestations. ${ }^{1,3,5,6}$ Sjögren's syndrome is not curable and typically is not diagnosed until irreversible organ damage has occurred. ${ }^{1,5}$ The disease affects 3.1 million North Americans, including approximately 430,000 Canadians, with 90\% of those affected being women. ${ }^{1,7}$ The prevalence of primary SS in China has been reported to be $0.77 \%^{8}$ and in the United Kingdom to be $0.1 \% .{ }^{9}$ Controversy exists regarding age of onset of the disease, with one report suggesting two peaks in life during which there is an increased risk of onset, at 20 to 30 years of age and after the mid-50s. ${ }^{10}$ However, others report that the average age of onset is 45 years. ${ }^{1,5}$

While patients with SS exhibit dry eye symptoms and signs, it is not possible to diagnose the condition using ocular symptoms and signs alone. The American-European Consensus Group developed a list of six criteria to help diagnose SS. ${ }^{6}$ For
\end{abstract}

Copyright 2015 The Association for Research in Vision and Ophthalmology, Inc www.iovs.org | ISSN: 1552-5783 positive diagnosis, at least four of six of the criteria must be met, with at least one of the four criteria met being positive for the autoantibodies Ro and/or La, or a positive salivary gland biopsy score ( $\geq 1$ focus score of $4 \mathrm{~mm}$ of tissue). More recently, the American College of Rheumatology has proposed an expert consensus approach to the diagnosis of SS, with revised considerations for positive diagnosis to at least two of three criteria. The three criteria include (1) positive serum anti-SSA/ Ro and/or anti-SSB/La, or (positive rheumatoid factor and ANA titer $\geq 1: 320$ ); (2) labial salivary gland biopsy exhibiting focal lymphocytic sialadenitis with a focus score $\geq 1$ focus $/ 4 \mathrm{~mm}$; and (3) keratoconjunctivitis sicca with ocular staining score $\geq 3$ (assuming that individual is not currently using daily eye drops for glaucoma and has not had corneal surgery or cosmetic eyelid surgery in the last 5 years). ${ }^{11}$ The revised classification criteria were not available at the time of this study and, thus, were not used for classification of SS.

Sjögren's syndrome has been classified as aqueous-deficient dry eye by the International Dry Eye Workshop, with much attention being paid to the lack of aqueous tear production. ${ }^{12}$ 
However, meibomian gland dysfunction also has been diagnosed in patients with SS. ${ }^{13}$

Meibomian glands are sebaceous glands located in the tarsal plates of the upper and lower eyelids. There are approximately 30 to 40 glands in the upper lid and 20 to 30 glands in the lower lid. ${ }^{14}$ These glands secrete the lipid layer of the tear film onto the surface of the eye, which helps prevent rapid evaporation of the tear film and subsequent ocular discomfort. ${ }^{14}$ Meibomian gland dysfunction (MGD) has been classified by the International Workshop on Meibomian Gland Dysfunction as a chronic, diffuse abnormality of the meibomian glands, commonly characterized by terminal duct gland obstruction and/or qualitative/quantitative changes in the glandular secretion. This may result in alteration of the tear film, symptoms of eye irritation, clinically apparent inflammation, and ocular surface disease. ${ }^{15}$ Meibomian gland dysfunction increases with age, contact lens wear, and androgen deficiency. ${ }^{16-19}$ This would suggest that the severe dry eye signs and symptoms seen in SS may be due to a lack of tear production and also poor tear quality, due to a reduced lipid layer. However, the degree of meibomian gland loss in SS patients requires further investigation.

Historically, the presence of MGD has been evaluated based on characteristics of secretion quality and quantity from the glands, as well as gland orifice plugging. Currently, userfriendly techniques are available to image the actual "dropout" of the meibomian glands from the upper and lower lids via the use of an infrared camera. ${ }^{20,21}$ Dropout of meibomian glands is considered areas of the lids that appear to have glands missing or have truncated glands, from areas that should histologically have meibomian glands. These areas of loss appear as dark areas on the images of the lids, compared to the white-colored area of the glands. To date, only one study has used the use of meibography to evaluate MGD in SS patients ${ }^{13}$ and to our knowledge, no studies have compared meibography to the measured lipid layer thickness (LLT) in such patients. Thus, the purpose of this study was to evaluate and compare meibomian gland dropout and LLT in patients with and without SS dry eye.

\section{MeTHODS}

Approval of this project was granted through the Office of Research Ethics at the University of Waterloo, and all procedures adhered to the Declaration of Helsinki. Participants were recruited at the Centre for Contact Lens Research and Ocular Health Clinic at the School of Optometry and Vision Science, University of Waterloo. Written informed consent was obtained from each participant after explanation of the purpose of the study and study procedures to be undertaken. A total of 21 participants completed the study. Current and past contact lens wearers and participants with lid abnormalities (e.g., stye, chalazion, trichiasis, or coloboma) that would interfere with lid eversion were excluded from the study.

The study was a one-visit study consisting of two parts: Part 1 consisted of a screening visit, including Ocular Surface Disease Index (OSDI) questionnaire, biomicroscopic assessment, staining assessment, followed by a break of 30 minutes; and part 2 included collection of primary outcome variables, LLT, lid parallel conjunctival folds (LIPCOF), noninvasive tear breakup time (NITBUT), meibomian gland expression, and meibography.

All assessments were carried out by the same examiner.

\section{Participant Categorization}

Participants were categorized into two groups: an SS group and a control group. Participants who were diagnosed previously with SS, who had symptoms of dry eye and discomfort for at least 3 months, and a staining score of $>4$ of 9 in at least one eye were placed into the SS group. Participants with no previous diagnosis of SS who reported an OSDI score of $\leq 12^{22,23}$ and who showed a staining score of $\leq 1$ of 9 for both eyes were included in the control group.

Those in the SS group also were asked questions regarding their dry eye and dry mouth symptoms. These questions are included in the American-European Consensus Group Criteria. $^{6}$

\section{Staining Assessment}

A grading scale adopted from the scales created by van Bijsterveld,${ }^{24}$ the Oxford grading scale, ${ }^{25}$ and members of the Sjögren's International Collaborative Clinical Alliance (SICCA $)^{26}$ was used to grade corneal and conjunctival cell staining. Sodium fluorescein dye (strips obtained from Flu Glo; Akorn, Inc., Lakeforest, IL, USA) was used to assess corneal staining. Staining of the conjunctiva was assessed after the instillation of lissamine green dye (strips obtained from Green Glo; Sigma Pharmaceuticals, Monticello, IA, USA). Three zones were graded for staining: the temporal conjunctiva, cornea, and nasal conjunctiva. Staining in each zone was graded accordingly: 0 , no staining; 1 , few separate spots; 2 , many separate spots; and 3, confluent spots. Conjunctival and corneal staining scores were combined, as proposed by the American-European Consensus Group Criteria for diagnosis of SS; thus, total scores for each eye ranged between 0 (no staining) and 9 (confluent staining in all zones).

\section{LLT Evaluation}

The tear film LLT was measured for each participant using the Keeler Tearscope Plus (Keeler Instruments, Inc., Broomall, PA, USA), as described previously. ${ }^{27-29}$ The lipid layer for each participant was graded by appearance using the system described by Guillon and Guillon. ${ }^{30}$ Lipid layer classification was given a quantitative value, ${ }^{30,31}$ for subsequent statistical analysis.

\section{Lid Parallel Conjunctival Folds (LIPCOF)}

The presence of LIPCOF was examined on the inferior temporal region of the conjunctiva along the lower lid margin just outside of the limbal region. ${ }^{32}$ The LIPCOF for each participant was graded using Hoh's grading scale (grade 0 , no permanently present fold; grade 1 , single, small fold, less than tear prism height; grade 2, multiple folds, up to the tear prism height; grade 3, multiple folds, higher than tear prism height). ${ }^{32-34}$

\section{Noninvasive Tear Breakup Time (NITBUT)}

The NITBUT was measured using the Atlas Corneal Topographer (Carl Zeiss Meditec Ag, Oberkochen, Germany). The participant was instructed to blink three consecutive times and then asked to hold his/her eyes open for as long as possible. The time elapsed between the last blink and first sign of distortion occurring of the ring pattern was recorded as the NITBUT. This was repeated three times for each eye and the average reported.

\section{Meibomian Gland Expressibility and Secretion Quality}

Obstruction of the meibomian gland orifices and quality of gland expression were evaluated using the gland evaluator 
TABLE. Average Scores and Respective $P$ Values for the Control and SS Groups for NITBUT, LIPCOF, LLT, Subjective Gland Dropout Scores, and Digital Gland Dropout Scores

\begin{tabular}{|c|c|c|c|}
\hline & $\begin{array}{l}\text { Control Group } \\
\text { Median (IQR) }\end{array}$ & $\begin{array}{c}\text { SS Group } \\
\text { Median (IQR) }\end{array}$ & $P$ Value \\
\hline OSDI, scale $0-100$ & $2.1(0.0-2.6)$ & $48.0(23.0-56.2)$ & $<0.001^{*}$ \\
\hline Obstruction of central 8 glands, \# of obstructed glands & $2.5(1.4-2.8)$ & $8.0(7.0-8.0)$ & $<0.001^{*}$ \\
\hline Quality of meibum, scale $0-3$ & $0.5(0.0-1.1)$ & $2.0(1.0-3.0)$ & $0.002^{*}$ \\
\hline Staining score, scale $0-9$ & $0.5(0.5-0.5)$ & $5.5(3.5-6.5)$ & $<0.001^{*}$ \\
\hline LIPCOF, grade $0-3$ & $2.0(0.9-2.1)$ & $2.5(2.0-3.0)$ & 0.2 \\
\hline NITBUT, sec & $9.5(6.4-17.6)$ & $3.7(2.5-4.2)$ & $<0.001^{*}$ \\
\hline LLT, $\mathrm{nm}$ & $60.0(45.0-100.0)$ & $15.0(15.0-15.0)$ & $0.001^{*}$ \\
\hline MG dropout, subjective grading, grade $0-3$, upper + lower totaling to 6 & $1.0(0.0-1.25)$ & $1.5(1.0-4.0)$ & $0.03^{*}$ \\
\hline MG dropout, digital grading, $0 \%-100 \%$ & $6.7(1.5-12.7)$ & $16.0(12.1-40.0)$ & $0.01^{*}$ \\
\hline
\end{tabular}

* Statistically significant.

developed by Korb et al. ${ }^{35}$ The device was designed to apply a constant force of $1.25 \mathrm{~g} / \mathrm{mm}^{2}$. The pressure was applied for approximately 12 to 15 seconds before the instrument was removed. The number of glands within central eight glands of the lower lid that did not express was recorded. Meibum that expressed from the glands was evaluated using a 0 to 3 scale: grade 0 , normal, clear oil expressed (cooking oil appearance); grade 1, opaque, diffusely turbid, normal viscosity; grade 2, opaque, increased viscosity; and grade 3, inspissated (i.e., toothpaste-like) or unable to express any material. ${ }^{36}$

\section{Meibography}

The meibomian glands were imaged using the infrared camera system of the Keratograph 4 (Oculus, Wetzlar, Germany), as described previously. ${ }^{20}$ Meibomian gland dropout was first evaluated subjectively using a 0 to 3 grading scale developed by Arita et al. ${ }^{17,36,37}$ (grade 0, no gland loss; grade 1, areas of gland loss $<33 \%$ of total gland area; grade 2 , area of gland loss between $33 \%-67 \%$ of total area; and grade 3, area of gland loss $>67 \%)$. Scores for the upper and lower eyelids were added, to obtain a total score (0-6) for each eye. Meibography images were analyzed using ImageJ (http://imagej.nih.gov/ij/; provided in the public domain by the National Institutes of Health, Bethesda, MD, USA), as described previously. ${ }^{20}$ During the subjective and digital analysis of gland dropout, the investigator was masked as to whether or not the participant had SS.

\section{Statistical Analysis}

Statistical analysis was conducted using Statistica 9 (StatSoft, Tulsa, OK, USA). Scores for measured variables were recorded as the combined average scores for both eyes. Differences in measured variables between groups were compared using the Mann-Whitney $U$ test. Correlation between LLT, NITBUT, and gland dropout was conducted using a Spearman rank correlation. A $P$ value of $<0.05$ was considered to be significant.

\section{Results}

There were 10 participants enrolled in the control group (three men and seven women; age $58.5 \pm 4.7$ years). Of the participants, 11 were enrolled in the SS group (1 man and 10 women; age $=56.0 \pm 9.1$ years). None of the control group and all 11 participants in the SS group reported the use of artificial tear drops. Along with artificial tears, other commonly reported methods to manage dryness symptoms among the SS participants included the use of warm compresses, lid scrubs, autologous serum, and cyclosporine eye drops, and omega-3 nutritional supplements.

The participants in the SS group had been diagnosed with SS from 4 to 13 years prior. All participants reported that dry eye symptoms preceded diagnosis of SS.

Median scores for the OSDI questionnaire, obstruction of the central eight glands of the lower lid, quality of meibum, staining scores, NITBUT, LIPCOF, LLT, gland dropout scores using subjective grading, and gland dropout scores using the digital grading are shown in the Table. Examples of images taken by the Keratograph 4 and used for further analysis are shown in the Figure.

There was a positive correlation between LLT and NITBUT $(r=0.54 ; P<0.05)$. There was a negative correlation between digital dropout scores and NITBUT $(r=-0.60, P<0.05)$. There was no significant correlation between the digital and subjective dropout scores with LLT.

\section{Discussion}

The results of this study indicate that patients with SS have a higher degree of MGD (poor quality, quantity, and morphological changes) than adults who do not experience dry eye. The presence and severity of MGD was evaluated by investigating meibomian gland obstruction, expressibility, dropout, as well as LLT and NITBUT. To our knowledge, this is the only study that has investigated LLT and gland dropout, and the relationship between the two in patients with SS. One other study by Shimazaki et al. ${ }^{13}$ investigated gland dropout in patients with SS and their findings support those from this study, in that gland dropout was significantly higher in the SS group. However, imaging of the gland dropout was not undertaken with a contemporary infrared system and there was no evaluation of LLT.

Historically, the dry eye experienced by people with SS has been thought to be due to severe aqueous deficiency, ${ }^{38}$ with little attention being paid to a possible role of evaporative dry eye. Meibomian gland dropout is the most common cause of evaporative dry eye. ${ }^{38-40}$ Dysfunction of the meibomian gland is characterized by stenosis or obstruction of the gland orifices on the lid margins, inflammatory changes (redness, edema, telangiectasia), as well as quantitative and qualitative changes to the secreted meibum. ${ }^{14}$ Stenosis of the gland orifices prevents meibum from being secreted from the glands. A build-up of meibum in the glands causes ductal dilation, cystic degeneration, loss of the meibocytes, and eventual gland atrophy. ${ }^{14} \mathrm{~A}$ reduction in meibum volume and reduced quality of meibum secreted onto the ocular surface 

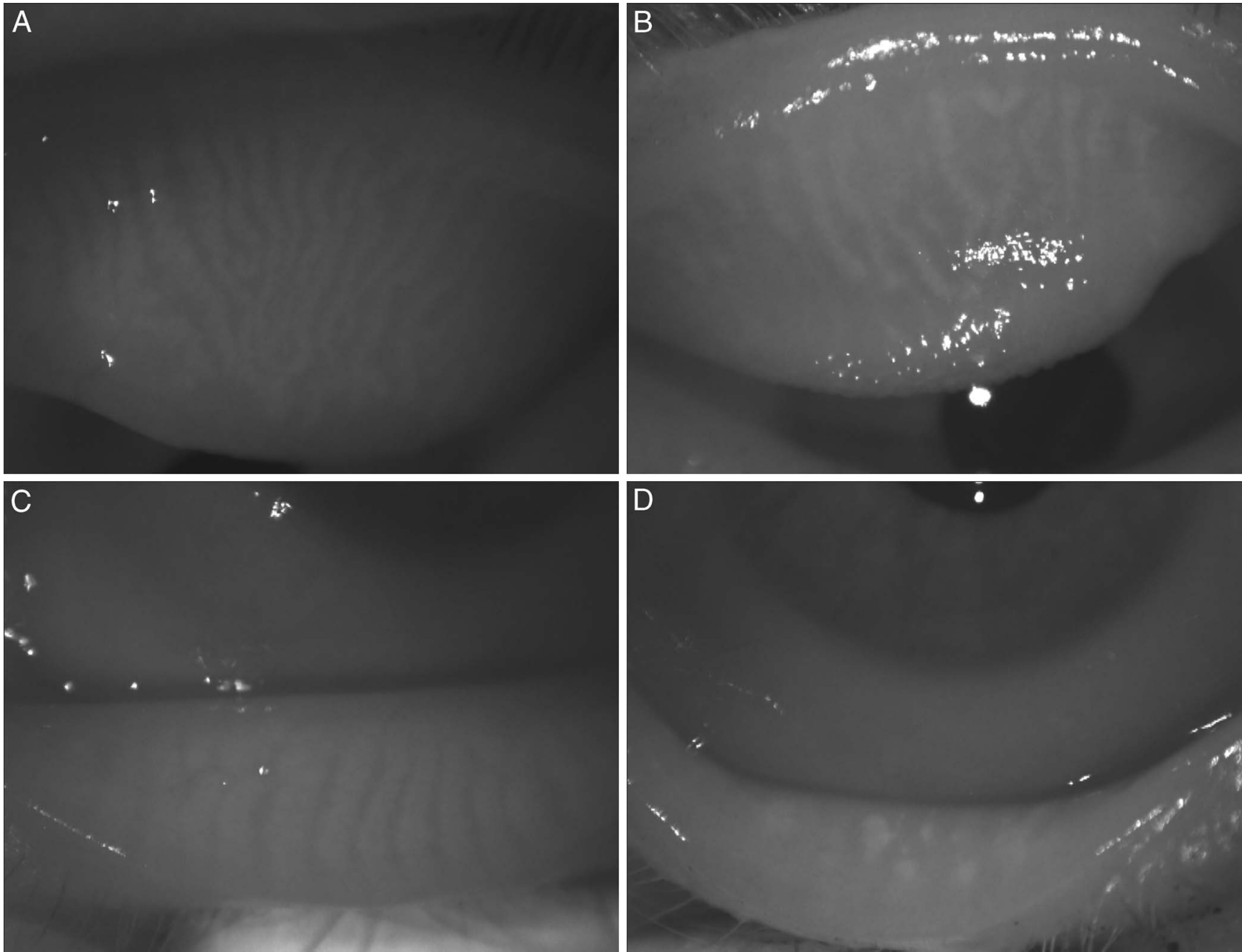

Figure. (A) Meibography image of the upper eyelid of a participant in the normal group with no MGD. (B) Meibography image of the upper eyelid of a participant in the SS group with MGD. (C) Meibography image of the lower eyelid of a participant in normal group with no MGD. (D) Meibography image of the lower eyelid of a participant in SS group with MGD.

leads to alterations and thinning of the lipid layer and increased evaporation of the tear film. ${ }^{14}$ Alteration to the lipid layer and LLT was evaluated in this study with the Tearscope. The Tearscope permitted viewing of the tear film lipid layer over the entire cornea, thus allowing evaluation of the lipid layer in an undisturbed state. Past studies have used the Tearscope to evaluate the lipid layer of potential and current contact lens wearers, to determine if the lipid layer would be suitable for lens wear, as well as to evaluate any changes that occur to the thickness and stability of the lipid layer during lens wear. ${ }^{27,28}$ The studies found that a thick homogenous lipid layer, characterized as "amorphous," tended to be the most stable. ${ }^{28}$ In this study, the participants in the SS group presented with a significantly thinner lipid layer, with the majority of the lipid layers characterized as being an "open meshwork." The positive correlation between LLT and NIBUT, with a thinner lipid layer being correlated positively with a shorter NITBUT, follows the trend previously reported by Maissa and Guillon. ${ }^{29}$ The shorter NITBUT and thin LLT found in the SS group could be explained further by the reduced quality of secreted meibum in the SS group.
This study not only evaluated changes to the lid margin and meibomian secretions, but also investigated the dropout of the glands in the tarsal plates. Imaging meibomian glands in the control and SS group was conducted successfully by using the Keratograph 4 , which has been used in previous work. ${ }^{20}$ The participants in the SS group had a higher degree of meibomian gland dropout, which likely contributed the thinner LLT and shorter NITBUT, compared to individuals in the control group. Our findings of more severe MGD and quicker evaporation rates in participants with SS are in agreement with previous studies. ${ }^{13,41}$

The subjective grading of meibomian gland dropout was higher for the SS group compared to the control group. Subjective grading has classically been used to evaluate gland dropout. The 0 to 3 grading scale used by Arita et al. ${ }^{17,36,37}$ was used in this study. A digital grading system reported by Srinivasan et al. ${ }^{20}$ also was used to further evaluate the area of functional meibomian glands which also demonstrated significantly higher dropout in the SS group compared to the control group.

The mechanism of increased MGD found in SS to date has not been extensively investigated. A study conducted by Villani et al. ${ }^{42}$ investigated the histopathology of meibomian 
glands in patients with primary and secondary SS using in vivo confocal microscopy. The results showed signs of inflammation of the meibomian glands that could be responsible for increased MGD. Other research groups have investigated the role of reduced androgen levels with increased MGD. ${ }^{43,44}$ Krenzer et al. ${ }^{16}$ investigated whether or not participants using antiandrogen therapy would develop MGD. The results showed a decrease in tear breakup time, an increase in gland orifice obstruction, and a decrease in the quality of the meibomian secretions. These results suggest that the drug therapy caused androgen deficiency that ultimately led to MGD. ${ }^{16}$ Sullivan et al. ${ }^{45}$ investigated whether patients with SS were androgen deficient, which may result in meibomian gland dysfunction. In their study, the serum concentrations of androgen precursors, active androgens, and androgen metabolites were measured in 22 postmenopausal women with SS, 10 with primary SS, and 12 with secondary SS. Results showed a decrease in androgen levels compared to normal controls that were age-matched, confirming that patients with SS were, indeed, androgen deficient. Although the results of these studies aid in the explanation of the increased MGD of participants with SS in this current study, serum androgen levels were not collected in this study nor were the presence of inflammatory markers in the meibomian glands, and, thus, it is difficult to conclude that reduced androgen levels and inflammation contributed to the observed increase in MGD.

It was interesting to note no differences between LIPCOF values between the two groups, as it has been suggested to be a valued determinant of dry eye. ${ }^{46}$ It has been shown that the degree of LIPCOF intensity is significantly higher with advancing age and more severe subjective symptoms. ${ }^{47,48}$ However, this was not the case in this study, as there were significantly different subjective symptoms between groups but similar LIPCOF values. The lack of significance between the two groups may have been due to the small sample size for each group, thus, more investigation with a larger sample size is required to determine factors contributing to LIPCOF.

We recognize that there are a number of limitations to this pilot study. This includes enrolling participants in the SS group who had been diagnosed with either primary or secondary SS and it is possible that systemic diseases in the participants who had secondary SS may have contributed to the meibomian gland loss. This warrants further investigation. In addition, the amount of time elapsed since diagnosis was not controlled for in this study. A longer duration of the disease, as well as a long time to diagnosis, may have contributed to additional meibomian gland loss. Thus, future studies on SS should perhaps consider only primary SS as an inclusion criterion, as well as analyze onset of symptoms and time since diagnosis. The LLT in this study was assessed using the Tearscope, which allowed categorization of the LLT according to the pattern observed. Although this provides an indication of the corresponding range of thickness, it does not provide the exact measurement of the thickness of the lipid layer and further studies are required to obtain accurate measurements of LLT in SS patients. Another limitation to this study was not including a group of participants with non-SS dry eye. Thus, it is difficult for us to conclude with certainty that the increased MGD seen in the SS group was specifically due to SS, rather than a contribution of the dry eye symptoms found in all people suffering from dry eye. Thus, it would be prudent for another study to be conducted including a control group of participants with non-SS dry eye.

In conclusion, this study showed that patients with SS showed higher meibomian gland dropout scores and reduced LLT and NITBUT, which likely contributed to the severe dry eye symptoms reported by SS dry eye patients. This study further supported previous work suggesting that dysfunction of the meibomian glands has an essential role in severe dry eye. This study also exemplifies the use of meibography for an easy visualization and assessment of the degree of gland dropout in patients with dry eye. Although the digital grading system is time-consuming in a clinical setting, the subjective method for analyzing the physical loss of meibomian glands could aid in a better understanding and management of the disease.

\section{Acknowledgments}

Supported by the Sjogren's Society of Canada.

Disclosure: K.L. Menzies, None; S. Srinivasan, None; C.L. Prokopich, None; L. Jones, None

\section{References}

1. Jonsson R, Vogelsang P, Volchenkov R, Espinosa A, WahrenHerlenius M, Appel S. The complexity of Sjogren's syndrome: novel aspects on pathogenesis. Immunol Lett. 2011;141:1-9.

2. Tabbara KF, Vera-Cristo CL. Sjogren syndrome. Curr Opin Opbthalmol. 2000;11:449-454.

3. Caffery B, Simpson T, Wang S, et al. Factor analysis of the clinical characteristics of primary Sjögren syndrome. Optom Vis Sci. 2010;87:742-750.

4. Nikolov NP, Illei GG. Pathogenesis of Sjögren's syndrome. Curr Opin Rbeumatol. 2009;21:465-470.

5. Bjerrum K, Prause JU. Primary Sjögren's syndrome: a subjective description of the disease. Clin Exp Rbeumatol. 1990;8:283-288.

6. Vitali C, Bombardieri S, Jonsson R, et al. Classification criteria for Sjögren's syndrome: a revised version of the European criteria proposed by the American-European Consensus Group. Ann Rbeum Dis. 2002;61:554-558.

7. Goransson LG, Haldorsen K, Brun JG, et al. The point prevalence of clinically relevant primary Sjögren's syndrome in two Norwegian counties. Scand J Rbeumatol. 2011;40: 221-224

8. Zhang NZ, Shi CS, Yao QP, et al. Prevalence of primary Sjögren's syndrome in China. J Rbeumatol. 1995;22:659-661.

9. Bowman SJ, Ibrahim GH, Holmes G, Hamburger J, Ainsworth JR. Estimating the prevalence among Caucasian women of primary Sjögren's syndrome in two general practices in Birmingham, UK. Scand J Rbeumatol. 2004;33:39-43.

10. Fox RI. Sjögren's syndrome. Lancet. 2005;366:321-331

11. Shiboski SC, Shiboski CH, Criswell L, et al. American College of Rheumatology classification criteria for Sjögren's syndrome: a data-driven, expert consensus approach in the Sjögren's International Collaborative Clinical Alliance cohort. Arth Care Res. 2012;64:475-487.

12. Lemp MA, Foulks GN. The definition and classification of dry eye disease: report of the Definition and Classification Subcommittee of the International Dry Eye WorkShop (2007). Ocular Surf. 2007;5:75-92.

13. Shimazaki J, Goto E, Ono M, Shimmura S, Tsubota K. Meibomian gland dysfunction in patients with Sjögren's syndrome. Opbthalmology. 1998;105:1485-1488.

14. Knop E, Knop N, Millar T, Obata H, Sullivan DA. The international workshop on meibomian gland dysfunction: report of the subcommittee on anatomy, physiology, and pathophysiology of the meibomian gland. Invest Opbthalmol Vis Sci. 2011;52:1938-1978.

15. Nelson JD, Shimazaki J, Benitez-del-Castillo JM, et al. The international workshop on meibomian gland dysfunction: 
report of the definition and classification subcommittee. Invest Ophthalmol Vis Sci. 2011;52:1930-1937.

16. Krenzer KL, Dana MR, Ullman MD, et al. Effect of androgen deficiency on the human meibomian gland and ocular surface. J Clin Endocrinol Metab. 2000;85:4874-4882.

17. Arita R, Itoh K, Inoue K, Kuchiba A, Yamaguchi T, Amano S. Contact lens wear is associated with decrease of meibomian glands. Ophthalmology. 2009;116:379-384.

18. Borchman D, Foulks GN, Yappert MC, et al. Physical changes in human meibum with age as measured by infrared spectroscopy. Ophthalmic Res. 2010;44:34-42.

19. Den S, Shimizu K, Ikeda T, Tsubota K, Shimmura S, Shimazaki J. Association between meibomian gland changes and aging, sex, or tear function. Cornea. 2006;25:651-655.

20. Srinivasan S, Menzies K, Sorbara L, Jones L. Infrared imaging of meibomian gland structure using a novel keratograph. Optom Vis Sci. 2012;89:788-794.

21. Srinivasan S, Menzies KL, Sorbara L, Jones L, Sickenberger W. Meibography of the upper lid. Optician. 2011;242:12-13.

22. Miller KL, Walt JG, Mink DR, et al. Minimal clinically important difference for Ocular Surf disease index. Arch Ophthalmol. 2010;128:94-101.

23. Srinivasan S, Joyce E, Boone A, Simpson T, Jones L, Senchyna M. Tear lipocalin and lysozyme concentrations in postmenopausal women. Ophthalmic Phys Optics. 2010;30:257-266.

24. van Bijsterveld OP. Diagnostic tests in the Sicca syndrome. Arch Ophthalmol. 1969;82:10-14.

25. Bron AJ, Evans VE, Smith JA. Grading of corneal and conjunctival staining in the context of other dry eye tests. Cornea. 2003;22:640-650.

26. Whitcher JP, Shiboski CH, Shiboski SC, et al. A simplified quantitative method for assessing keratoconjunctivitis sicca from the Sjogren's Syndrome International Registry. Am J Ophthalmol. 2010;149(3:405-415.

27. Guillon JP. Noninvasive Tearscope Plus routine for contact lens fitting. Cont Lens Anterior Eye. 1998;21(suppl 1):S31-S40.

28. Guillon M, Styles E, Guillon JP, Maissa C. Preocular tear film characteristics of nonwearers and soft contact lens wearers. Optom Vis Sci. 1997;74:273-279.

29. Maissa C, Guillon M. Tear film dynamics and lipid layer characteristics-effect of age and gender. Cont Lens Anterior Eye. 2010;33:176-182.

30. Guillon JP, Guillon M. The role of tears in contact lens performance and its measurement. In: Ruben M, Guillon M, eds. Contact Lens Practice. London: Chapman and Hall; 1994: 452-483.

31. Rae SM, Price HC. The effect of soft contact lens wear and time from blink on wavefront aberration measurement variation. Clin Exp Optom. 2009;92:274-282.

32. Pult H, Sickenberger W. LIPCOF and contact lens wearers: a new tool of forecast subjective dryness and degree of comfort of contact lens wearers. Contactologia. 2000;22:74-79.
33. Pult H, Murphy PJ, Purslow C. A novel method to predict the dry eye symptoms in new contact lens wearers. Optom Vis Sci. 2009;86:E1042-E1050.

34. Pult H, Purslow C, Berry M, Murphy PJ. Clinical tests for successful contact lens wear: relationship and predictive potential. Optom Vis Sci. 2008;85:E924-E929.

35. Korb DR, Blackie CA. Meibomian gland diagnostic expressibility: correlation with dry eye symptoms and gland location. Cornea. 2008;27:1142-1147.

36. Arita R, Itoh K, Maeda S, et al. Proposed diagnostic criteria for obstructive meibomian gland dysfunction. Ophthalmology. 2009;116:2058-2063.

37. Arita $\mathrm{R}$, Itoh $\mathrm{K}$, Inoue $\mathrm{K}$, Amano $\mathrm{S}$. Noncontact infrared meibography to document age-related changes of the meibomian glands in a normal population. Ophthalmology. 2008; 115:911-915.

38. Lemp MA. Research in dry eye: report of the Research Subcommittee of the International Dry Eye WorkShop (2007). Ocular Surf. 2007;5:179-193.

39. Bron AJ, Tiffany JM. The contribution of meibomian disease to dry eye. Ocular Surf. 2004;2:149-165.

40. Bron AJ, Tiffany JM, Gouveia SM, Yokoi N, Voon LW. Functional aspects of the tear film lipid layer. Exp Eye Res. 2004;78:347360.

41. Goto E, Matsumoto Y, Kamoi M, et al. Tear evaporation rates in Sjögren's syndrome and nonSjogren dry eye patients. Am J Ophthalmol. 2007;144:81-85.

42. Villani E, Beretta S, De Capitani M, Galimberti D, Viola F, Ratiglia R. In vivo confocal microscopy of meibomian glands in Sjögren's syndrome. Invest Ophthalmol Vis Sci. 2011;52:933939.

43. Sullivan DA, Sullivan BD, Ullman MD, et al. Androgen influence on the meibomian gland. Invest Ophthalmol Vis Sci. 2000;41:3732-3742.

44. Sullivan DA, Wickham LA, Rocha EM, et al. Androgens and dry eye in Sjögren's syndrome. Ann N Y Acad Sci. 1999;876:312324.

45. Sullivan DA, Belanger A, Cermak JM, et al. Are women with Sjögren's syndrome androgen-deficient? J Rheumatol. 2003; 30:2413-2419.

46. Schirra F, Hoh H, Kienecker C, Ruprecht KW. Using LIPCOF (lid-parallel conjunctival fold) for assessing the degree of dry eye, it is essential to observe the exact position of that specific fold. Adv Exp Med Biol. 1998;438:853-858.

47. Nemeth J, Fodor E, Lang Z, et al. Lid-parallel conjunctival folds (LIPCOF) and dry eye: a multicentre study. Br J Ophthalmol. 2012;96:1380-1385.

48. Zhang X, Li Q, Zou H, et al. Assessing the severity of conjunctivochalasis in a senile population: a community-based epidemiology study in Shanghai, China. BMC Pub Health. 2011;11:198. 